\title{
Incidencia del capital intelectual en el rendimiento financiero de las Universidades de Chile
}

\section{Incidence of intellectual capital in the financial performance of universities of Chile}

\author{
Carlos Galleguillos Cortés ${ }^{1,3} \quad$ José Luis Silva Muna ${ }^{2 *} \quad$ Pablo Becerra Muñoz ${ }^{3}$
}

Recibido 18 de Noviembre de 2016, aceptado 26 de Diciembre de 2017

Received: November 18, 2016, Accepted: December 26, 2017

\begin{abstract}
RESUMEN
El objetivo del artículo es investigar sobre la incidencia de las variables que componen el Capital Intelectual en el rendimiento financiero de las Instituciones de Educación Superior, específicamente en las universidades de Chile. Fueron investigadas cincuenta y siete universidades entre públicas y privadas, de las cuales se obtuvo ratios financieros y las dimensiones de Capital Intelectual, es decir, Capital Humano, Estructural y Relacional. Los resultados obtenidos apoyan parcialmente las hipótesis planteadas las cuales indican que el Capital Humano, Relacional y Estructural contribuyen al desempeño financiero que tienen las Universidades.
\end{abstract}

Palabras clave: Desempeño financiero, capital intelectual, capital humano, capital estructural, capital relacional.

\begin{abstract}
The target of this article is to investigate the incidence of variables that compose Intellectual Capital in the financial performance of Institutions of Higher Education, specifically at the universities of Chile. Fifty-seven public and private universities were investigated, from which financial ratios and the dimensions of Intellectual Capital were obtained, i.e., Human, Structural and Relational Capital. The results obtained partially support the hypotheses that indicate that Human, Relational and Structural Capital contributes to the financial performance that the Universities have.
\end{abstract}

Keywords: Financial performance, intellectual capital, human capital, structural capital, relational capital.

\section{INTRODUCCIÓN}

Las instituciones públicas de educación superior representan un área interesante de investigación porque se consideran actores clave en la sociedad del conocimiento y están en el centro de la agenda política en el ámbito nacional e internacional.
En efecto, el desempeño organizacional en las instituciones públicas de educación superior cobra relevancia, debido al papel fundamental que estas organizaciones tienen en la formación de capital humano, que sustenta el desarrollo económico de los países, por la competencia que representa el incremento de instituciones públicas y privadas, la

\footnotetext{
1 Universidad de Santiago de Chile. Facultad de Administración y Economía, Departamento de Administración, Santiago. e-mail: carlos.galleguillos@usach.cl

2 Universidad de Atacama, Facultad de Ingeniería, Departamento de Ingeniería Comercial, Copiapó. e-mail: jose.silva@uda.cl

3 Universidad de Atacama, Facultad de Ingeniería, Departamento de Industria y Negocios, Copiapó. e-mail: carlos.galleguillos@uda.cl; pablo.becerra@uda.cl

* Autor de correspondencia: jose.silva@uda.cl
} 
presión gubernamental para demostrar su rendimiento y la transparencia en la rendición de cuentas a la sociedad.

En este sentido, el estudio de los recursos intangibles es un tema central dado que contribuye a mejorar las capacidades y a generar valor en las organizaciones. Específicamente, el Capital Intelectual [1].

Según Sullivan [2], el Capital Intelectual es el principal determinante de la competitividad, principalmente por la creciente importancia del conocimiento y la economía basada éste, los patrones cambiantes de las actividades interpersonales, la sociedad en red y la aparición de la innovación.

De esta manera, es de vital importancia para las organizaciones entender cómo se crea, gestiona, mide y se evalúa, el Capital Intelectual [3]. Sin embargo, existe evidencia en la literatura como la de Guthrie, Ricceri y Dumay [4], quienes aducen que el sector público es una de las áreas menos abordadas por la investigación del Capital Intelectual.

Por tanto, parece relevante determinar la influencia de las dimensiones del Capital Intelectual en el rendimiento financiero de Instituciones de Educación Superior.

\section{BREVE REVISIÓN DE LA LITERATURA}

El reconocimiento mundial del Capital Intelectual ha permitido confirmar a ésta, como una disciplina académica [5]. Esta disciplina ha sido estudiada ampliamente y sus fundamentos se explican en cuanto a que ha permitido un acuerdo sobre el efecto positivo que tiene éste en la creación de valor dentro de las organizaciones [6 - 10].

En esta década, se ha continuado fundamentando que no basta con gestionar los elementos tangibles como la maquinaria, edificios, instalaciones, depósitos en bancos, entre otros. Cada vez más, los elementos intangibles como el conocimiento, las habilidades, valores, actitudes y otros elementos son los que marcan el diferencial de competitividad de una organización [11].

Es así, como se ha ido fortaleciendo el concepto de Capital Intelectual, relacionándose con la posesión de conocimientos, experiencia aplicada, tecnología organizacional, relaciones con clientes y destrezas profesionales [7]. Así también, con la creación de ventaja competitiva y en la generación de valor, dinamizados por una integración de diferentes activos intangibles basada en flujos de conocimiento o en actividades intelectuales de la organización.

\section{RELACIÓN DE LAS DIMENSIONES DEL CAPITAL INTELECTUAL EN LAS UNIVERSIDADES}

En el contexto de las universidades, las dimensiones del capital intelectual son las siguientes:

El Capital Humano: Constituye el conjunto de conocimientos, habilidades y actitudes de los empleados los cuales contribuyen a crear bienes tangibles e intangibles. Es decir, incluye la experiencia, conocimientos y experiencias de investigadores, profesores, personal técnico, estudiantes y personal administrativo [12]. Por otra parte, Sveiby [10] define el Capital Humano como la capacidad de actuar en una amplia variedad de situaciones para crear bienes tangibles e intangibles.

El Capital Relacional: Es la capacidad de una organización para crear valor en la interacción con sus grupos de interés externos. Es decir, son los recursos intangibles y capacidades de generar valor vinculado a las relaciones internas y externas de la Universidad. Esto, incluye sus relaciones con los socios privados, la posición y la imagen en las redes, su prestigio académico, su asociación con el sector empresarial y los gobiernos regionales, sus vínculos con organizaciones sin fines de lucro y la sociedad civil en general, colaboraciones con centros internacionales de investigación, redes y alianzas, entre otros [12].

El Capital Estructural: Corresponde al valor del conocimiento creado en la organización. Conocimiento que ha sido sistematizado, explícito o interiorizado y que es propiedad de la organización [13]. Secundo [12] aduce que son las capacidades de una organización en términos de estructura organizacional, procedimientos, rutinas, sistemas, hardware, bases de datos y las culturas organizacionales. Esto, se refleja en las Universidades a partir de invenciones, procesos, derechos de autor, patentes, tecnologías, estrategia, sistemas, entre otros. 


\section{RELACIÓN ENTRE EL CAPITAL INTELECTUAL Y SU IMPACTO EN EL DESEMPEÑO ORGANIZACIONAL}

En la actualidad, existe una tendencia a que las organizaciones públicas introduzcan procesos de gestión del sector privado [14 - 15].

En este sentido, el uso del Balance Scorecard de Kaplan y Norton [16], ha ofrecido una explicación sobre como las medidas financieras tradicionales son complementadas con medidas operativas orientadas hacia los clientes, procesos internos y aprendizaje $\mathrm{y}$ actividades de crecimiento. Esta última, es la que se vincula con el Capital Intelectual a partir de tres categorías de activos intangibles esenciales: a) Capital Humano, corresponde a las destrezas, el talento y conocimiento de los empleados de una empresa; $b$ ) Capital de Información como las bases de datos, sistemas de información, redes e infraestructura tecnológica de una empresa, y finalmente; c) Capital Organizacional, que hace alusión a la cultura de la empresa, su liderazgo, la alineación de su personal con los objetivos estratégicos y la habilidad de los empleados para compartir el conocimiento. Por tanto, la gestión del Capital Intelectual para las universidades se basa en la premisa de que la producción y difusión del conocimiento puede contribuir en el desempeño financiero.

La necesidad de que las universidades tengan una mayor participación con su comunidad en general y la preocupación de asegurar comparaciones y benchmarking entre ellas, hace necesaria la gestión y divulgación de información sobre Capital Intelectual.

\section{METODOLOGÍA}

\section{Objetivo}

Determinar la incidencia de las dimensiones del Capital Intelectual en el rendimiento financiero de las Instituciones de Educación Superior, específicamente en las Universidades de Chile.

\section{Modelo}

Se propone un modelo de regresión lineal múltiple con el cual se espera probar las hipótesis respectivas. El modelo queda representado en la siguiente forma (1).

$$
\begin{gathered}
(\mathrm{ROS})=\mathrm{b}_{0}+\mathrm{b}_{1} \mathrm{X}_{1}+\mathrm{b}_{2} \mathrm{X}_{2}+\mathrm{b}_{3} \mathrm{X}_{3}+\beta_{4} \mathrm{X}_{4}+\beta_{5} \mathrm{X}_{5}+ \\
\beta_{6} \mathrm{X}_{6}+\beta_{7} \mathrm{X}_{7}+\mathrm{e}
\end{gathered}
$$

Donde:

$\beta=$ Coeficiente

$\mathrm{e}=$ Error

$\mathrm{X}_{1}=\mathrm{N}^{\circ}$ de académicos

$\mathrm{X}_{2}=\mathrm{N}^{\circ}$ de académicos con Jornada Completa

Equivalente (JCE)

$\mathrm{X}_{3}=\mathrm{N}^{\circ}$ de doctores

$\mathrm{X}_{4}=$ Años de acreditación

$\mathrm{X}_{5}=\mathrm{N}^{\circ}$ de programas acreditados

$\mathrm{X}_{6}=$ Proyectos Fondecyt

$\mathrm{X}_{7}=\mathrm{N}^{\circ}$ publicaciones WOS + Scielo

Basados en estos conceptos, podemos representar tales indicadores con relación a las respectivas dimensiones del Capital Intelectual en la Tabla 1. En la dimensión de Capital Relacional, se utilizan los indicadores $\mathrm{X}_{4}$ y $\mathrm{X}_{5}$ debido a la capacidad de éstos para crear valor. Lo anterior, en relación con sus grupos de interés externos como son el prestigio, el reconocimiento o la marca.

Tabla 1. Dimensiones del Capital Intelectual.

\begin{tabular}{|l|l|}
\hline $\begin{array}{l}\text { Dimensiones Capital } \\
\text { Intelectual }\end{array}$ & Indicadores que explican la dimensión \\
\hline Capital Humano & $\begin{array}{l}\mathrm{X}_{1}: \mathrm{N}^{\circ} \text { de académicos } \\
\text { Completa Equivalente (JCE) } \\
\mathrm{X}_{3}: \mathrm{N}^{\circ} \text { de doctores }\end{array}$ \\
\hline Capital Relacional & $\begin{array}{l}\mathrm{X}_{4}: \text { Años de acreditación } \\
\mathrm{X}_{5}: \mathrm{N}^{\circ} \text { de programas acreditados }\end{array}$ \\
\hline Capital Estructural & $\begin{array}{l}\mathrm{X}_{6}: \text { Proyectos Fondecyt } \\
\mathrm{X}_{7}: \mathrm{N}^{\circ} \text { publicaciones WOS + Scielo }\end{array}$ \\
\hline
\end{tabular}

\section{Hipótesis}

Las hipótesis que se desea probar son las siguientes:

H1: Existe una relación positiva y significativa entre el capital humano y el rendimiento financiero de las Instituciones de Educación Superior.

H2: Existe una relación positiva y significativa entre el capital relacional y el rendimiento financiero de las Instituciones de Educación Superior.

H3: Existe una relación positiva y significativa entre el capital estructural y el rendimiento financiero de las Instituciones de Educación Superior. 


\section{Muestra}

Se analizan cincuenta y siete de las sesenta y un Universidades Chilenas (públicas y privadas) para el período 2014. Los datos se obtuvieron del Servicio de Información de Educación Superior (SIES), perteneciente al Ministerio de Educación.

\section{Análisis}

El modelo se analiza a partir de una regresión lineal múltiple la cual permite contrastar el nivel de significancia de las variables determinadas.

Finalmente, los cálculos de análisis multivariable fueron realizados a través del Software Stata 14.

\section{RESULTADOS}

Los resultados de este artículo son expresados en distintas tablas que se presentan a continuación.

En la Tabla 2 queda reflejado el resumen estadístico de las variables de estudio.

En general, el modelo propuesto explica la relación con un R-Squared $=0.1406$ y un bajo índice de correlación. Esto, se visualiza en la Tabla 3.

Por otro lado, los valores de los coeficientes se expresan en la Tabla 4, siendo expresados éstos en tanto por uno. El número de académicos JCE ha demostrado ser positivo y significativo al nivel de 0,05 ( $\mathrm{P}<0,05)$, con un coeficiente de 0,0001 . Esto, quiere decir que el aumento en una unidad de los académicos JCE contribuye en un aumento de $0,01 \%$ en el ROS.

En esta misma dirección, el número de áreas de acreditación ha demostrado ser positivo y significativo al nivel de $0,1(\mathrm{P}<0,1)$, con un coeficiente de 0,03 . Esto, quiere decir que un aumento en una unidad de las áreas de acreditación tiene un impacto de un 3\% en el ROS.

Los proyectos FONDECYT y el número de publicaciones WOS y SCIELO ha demostrado ser positivo y significativo al nivel de $0,1(\mathrm{P}<0,1)$, con un coeficiente de 0,004 . Esto, quiere decir que el aumento en una unidad de los proyectos FONDECYT y el número de publicaciones WOS y SCIELO, debería contribuir a aumentar el ROS en $0,4 \%$.
Por lo tanto, parcialmente los resultados de las hipótesis propuestas, relacionados a los indicadores de capital humano, capital relacional y el capital estructural sobre el ROS, han demostrado ejercer un efecto estadístico significativo.

Tabla 2. Resumen estadístico de las variables.

\begin{tabular}{|c|c|c|c|c|c|}
\hline variable & obs & Mean & std. Dev. & Min & $\max$ \\
\hline 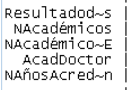 & $\begin{array}{l}57 \\
57 \\
57 \\
57 \\
57\end{array}$ & $\begin{array}{l}0438733 \\
1215.754 \\
530.9047 \\
169.9825 \\
3.438596\end{array}$ & $\begin{array}{l}0838937 \\
1072.338 \\
482.3425 \\
235.8426 \\
2.187771\end{array}$ & $\begin{array}{r}-.3224896 \\
26 \\
8.477273 \\
1 \\
0\end{array}$ & $\begin{array}{r}.2268152 \\
411 \\
2085.83 \\
1223 \\
7\end{array}$ \\
\hline $\begin{array}{l}\text { Náreasacre n } \\
\text { ProyectosF } \sim t \\
\text { Pub icacio s }\end{array}$ & $\begin{array}{l}57 \\
57 \\
57\end{array}$ & $\begin{array}{l}2.561404 \\
9.894377 \\
180.7544\end{array}$ & & $\begin{array}{l}0 \\
0 \\
0\end{array}$ & $\begin{array}{r}5 \\
125 \\
2028\end{array}$ \\
\hline
\end{tabular}

Tabla 3. Correlación de las variables.

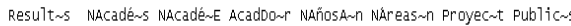

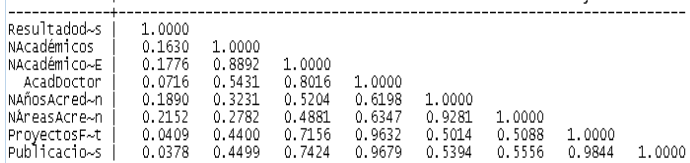

Tabla 4. Resultados modelo regresión lineal múltiple.

\begin{tabular}{|c|c|c|c|c|c|c|}
\hline Resultadode1Ejer $\sim 5$ & coef. & $\begin{array}{l}\text { Robust } \\
\text { std. Err. }\end{array}$ & $\mathrm{t}$ & $P>|t|$ & [95\% conf. & Interval] \\
\hline 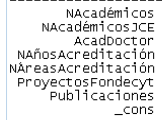 & $\begin{array}{r}-.000023 \\
.0001503 \\
-.0003079 \\
-.009576 \\
.000293 \\
-.0007626 \\
-.0002606 \\
.0003696\end{array}$ & $\begin{array}{l}.0000211 \\
.0000764 \\
.0002645 \\
.01017122 \\
.016489 \\
.0025742 \\
.0001578 \\
.033367\end{array}$ & $\begin{array}{r}-1.09 \\
1.97 \\
-1.16 \\
-0.89 \\
1.83 \\
1.85 \\
-1.65 \\
0.01\end{array}$ & $\begin{array}{l}0.282 \\
0.055 \\
0.250 \\
0.376 \\
0.074 \\
0.070 \\
0.105 \\
0.991\end{array}$ & $\begin{array}{l}-.0000654 \\
-3.35 e-06 \\
-00008395 \\
-.031103 \\
-.003008 \\
-0004111 \\
-.0000578 \\
-.0672867\end{array}$ & $\begin{array}{l}.0000194 \\
.0003039 \\
.0002237 \\
.011951 \\
.06060644 \\
.0099351 \\
.06005655 \\
.068259\end{array}$ \\
\hline
\end{tabular}

\section{DISCUSIÓN Y CONCLUSIONES}

El objetivo de esta investigación ha sido determinar la incidencia de las dimensiones del Capital Intelectual en el rendimiento financiero de las Instituciones de Educación Superior, específicamente en las Universidades de Chile. En este sentido, los resultados apoyan parcialmente las hipótesis propuestas entre una relación positiva y significativa entre el capital humano, capital relacional, capital estructural y el rendimiento financiero de las Instituciones de Educación Superior.

La gestión del Capital Intelectual, ha permitido contribuir a la creación de ventaja competitiva y en la generación de valor en las organizaciones, dinamizados por una integración de diferentes activos intangibles. Está, basada en flujos de conocimiento o en actividades intelectuales de la organización.

Diversos estudios, han demostrado la importancia de fortalecer las dimensiones del capital intelectual con el objeto de mejorar el desempeño. En este sentido y en función de los resultados obtenidos, se deduce que algunos indicadores del Capital Humano, 
Relacional y Estructural son aportes significativos a los resultados financieros de las Universidades en estudio. Lo anterior, es coherente con lo expresado en la revisión de la literatura, pues existen evidencias que respaldan la relación positiva entre capital intelectual y los resultados financieros.

Es importante entonces, señalar que un aumento en el número de académicos con JCE, áreas de acreditación, proyectos FONDECYT y publicaciones, contribuye a la mejora en el desempeño de las Universidades. Esto, como producto de haber realizado la medición en términos del resultado del ejercicio en función de los ingresos.

De acuerdo a la Tabla 2, existe una brecha importante entre el mínimo y máximo del Capital Humano, expresado en términos de académicos JCE y académicos doctores. Lo anterior, impactará probablemente en la obtención de proyectos FONDECYT y publicaciones científicas en las universidades con menos Capital Humano avanzado.

Así mismo, según el informe del Ministerio de Educación, los ingresos para las universidades están dado por: Aportes basales y fondos concursables, aranceles de pre y posgrado, donaciones, ingresos de cursos y programas de extensión, prestaciones de servicios y otros ingresos. De éstos, el $62 \%$ representan los aranceles de pre y post grado, reflejados éstos como los ingresos operacionales del sistema. En este sentido, el aumento de la matrícula en universidades acreditadas de 3 a 7 años, ha experimentado una tendencia al alza en el período 2005 - 2015 [17]. Probablemente, el aumento de la matrícula (por su relación directa con el ingreso operacional) sea dado por una positiva percepción de la calidad. Por lo tanto, si la calidad es un atributo de la satisfacción del cliente [18], el aumento de este último, puede ser ocasionado por una mejor actuación en el proceso de acreditación, por lo que en definitiva, puede repercutir en los ingresos percibidos. Dejando de esta manera, la duda sobre el real aporte de la variable matrícula (o ingreso, según como se desee considerar) en los resultados financieros.

Este modelo, presenta una importante limitación relacionada con el uso de datos de corte trasversal que imposibilita determinar la relación causal de las variables independientes respecto a las dependientes.
Así también, otro factor de imprecisión ha sido el hecho de no considerar otros indicadores del Capital Estructural como por ejemplo: La infraestructura, bibliotecas, laboratorios, entre otros.

Otra limitación que presenta la investigación se debe al hecho de que los datos utilizados son de corte trasversal. Esto, imposibilita establecer causalidad en la relación.

Finalmente, los resultados opuestos a los esperados en la confirmación de la hipótesis, tengan su explicación en la característica de los datos utilizados. Por lo cual se sugiere, en un próximo estudio cuantificar los indicadores de las dimensiones del Capital Humano, Relacional y Estructural en términos monetarios y no en unidades como ha sido realizado en este trabajo.

\section{REFERENCIAS}

[1] Y. Núñez y C. Rodríguez. "Gestión de recursos intangibles en Instituciones de Educación Superior". Revista de Administración de empresas. Sao Paulo, Vol. $55 \mathrm{~N}^{\circ}$ 1, pp. 65 - 77. 2015. ISSN 0034-7590. URL: http:// www.scielo.br/.pdf/rae/v55n1/0034-7590rae-55-01-0065.pdf. Fecha de consulta: 20 de Mayo de 2016. DOI: http://dx.doi. org/10.1590/S0034-759020150107.

[2] P. Sullivan. "Value driven Intellectual Capital: How to convert intangible corporate assets into market value". John Wiley \& Sons, Inc. 2000.

[3] H. Inkinen. "Review of empirical research on Intellectual Capital and firm performance". Journal of Intellectual Capital. Vol. $16 \mathrm{~N}^{\circ}$ 3, pp. 518 - 565. 2015.

[4] J. Guthrie, F. Ricceri and J. Dumay. "Reflections and projections: A decade of Intellectual Capital Accounting Research". The British Accounting Review. Vol. 44, Issue 2, pp. 68 - 82. 2012.

[5] A. Serenko and N. Bontis. "Investigating the current state and impact of the Intellectual Capital academic discipline". Journal of Intellectual Capital. Vol. $14 \mathrm{~N}^{\circ}$ 4, pp. 476 - 500. 2013.

[6] P. Drucker. "The rise of the knowledge society". Wilson Quarterly. Vol. 17 № 2, pp. 52-70. 1993.

[7] L. Edvinsson and M. Malone. "Intellectual Capital: Realising Your Company's True 
Value by Finding Its Hidden Brainpower". Harper Collins, New York, NY. 1997.

[8] R. Grant. "Toward a knowledge - based theory of the firm". Strategic Management Journal. Vol. 17, issue S2, pp. 109 - 122. 1996. DOI: $10.1002 / \mathrm{smj} .4250171110$

[9] T. Stewart. "Intellectual Capital: The New Wealth of Organizations". Doubleday, New York, NY. 1997.

[10] K. Sveiby. "The New Organizational Wealth: Managing and Measuring Knowledge - based Assets". Berrett - Koehlen, New York, NY. 1997.

[11] J. Daza, M. Wilches, L. Gómez, O. Durán y J. Cohen. "La gestión estratégica del Capital Intelectual en el entorno Latino Americano". Prospect. Vol. $8 \mathrm{~N}^{\circ}$ 2, pp. 31 - 36. 2010.

[12] G. Secundo, S. Elena, Z. Martinaitis and K. Leitner. "An intellectual capital maturity model (ICMM) to improve strategic management in European Universities: A dynamic approach. Journal of Intellectual Capital, Vol $16 \mathrm{~N}^{\circ}$ 2, pp. 419 - 442. 2015.
[13] M. Sánchez. "Breve inventario de los modelos para la gestión del conocimiento en las organizaciones". Acimed, Vol. $13 \mathrm{~N}^{\circ}$ 6. 2005.

[14] C. Hood. "A public management for all seasons?". Public Administration. Vol $69 \mathrm{~N}^{\circ}$ 1, pp. 3 - 19. 1991.

[15] M. Vienaz indiene and R. Ciarniene. "New public management: Theoretical and practical aspects". Engineering Economics. Vol. $5 \mathrm{~N}^{\circ}$ 55, pp. 44 - 50. 2007.

[16] R. Kaplan and D. Norton. "The Balance Scorecard-Measures that drive performance". Harvard Business Review. February, pp. 71 - 79. 1992.

[17] Consejo Nacional de Educación. Índice tendencia 2015, pp. 21 - 23. 2015.

[18] V. Zeithaml y M. Bitner. "Marketing de servicios: Un enfoque de integración del cliente a la empresa". 2002. 